\title{
Diallyl trisulfide sensitizes human melanoma cells to TRAIL-induced cell death by promoting endoplasmic reticulum-mediated apoptosis
}

\author{
MAYUMI MURAI ${ }^{1,2}$, TOSHIO INOUE ${ }^{1}$, MIKI SUZUKI-KARASAKI ${ }^{2}$, TOYOKO OCHIAI ${ }^{2}$, \\ CHISEI RA $^{1}$, SIGERU NISHIDA ${ }^{3}$ and YOSHIHIRO SUZUKI-KARASAKI ${ }^{1}$
}

\author{
${ }^{1}$ Division of Molecular Cell Immunology and Allergology, Department of Biomedical Sciences, Nihon University \\ School of Medicine; ${ }^{2}$ Department of Dermatology, Nihon University Surugadai Hospital; \\ ${ }^{3}$ Department of Chemistry, Nihon University School of Medicine, Tokyo, Japan
}

Received July 18, 2012; Accepted September 7, 2012

DOI: 10.3892/ijo.2012.1656

\begin{abstract}
Tumor necrosis factor-related apoptosis-inducing ligand (TRAIL) is promising for cancer treatment because of its selective cytotoxicity toward tumor cells. However, some cancer cell types including malignant melanoma cells are resistant to TRAIL cytotoxicity. Here, we show that diallyl trisulfide (DATS), a garlic organosulfur compound, sensitizes melanoma cells to TRAIL-induced apoptosis while sparing normal cells. DATS also potentiates apoptosis induced by agonistic antibodies against death receptors (DR) 4 and DR5. The amplification of DR-mediated apoptosis was associated with increased mitochondrial membrane potential collapse and caspase-3/7 activation. However, these events were not sufficient for full sensitization. TRAIL also induced endoplasmic reticulum (ER) stress, as indicated by the activation of X-box-binding protein 1 and caspase-12 and DATS potentiated both events. Moreover, inhibition of caspase-12, but not caspase-4, abolished the amplification of apoptosis, indicating that ER stress plays a crucial role. On the other hand, DATS and/or TRAIL induced minimal apoptosis and caspase-12 activation in melanocytes despite their substantial expression of DR4 and DR5 on the cell surface. Our data suggest that DATS amplifies death ligand-induced melanoma cell death by disrupting their adaptation to ER-mediated death pathway. The present findings raise the possibility that DATS may be combined with death ligands to treat TRAIL-resistance melanoma cells without impairing its tumor selectivity.
\end{abstract}

Correspondence to: Dr Yoshihiro Suzuki-Karasaki, Division of Molecular Cell Immunology and Allergology, Department of Biomedical Sciences, Nihon University School of Medicine, 30-1 Oyaguchikami-cho, Itabashi-ku, Tokyo 173-8610, Japan

E-mail: suzuki.yoshihiro@nihon-u.ac.jp

Key words: diallyl trisulfide, melanoma, tumor necrosis factorrelated apoptosis-inducing ligand, apoptosis, endoplasmic reticulum

\section{Introduction}

Malignant melanoma is the most deadly form of skin cancer. Once disseminated, the prognosis of patients with melanoma becomes very poor. The tumor is highly resistant to conventional chemotherapy, radiotherapy and immunotherapy, implying complexity and diversity of the factors controlling the disease. Alteration of the survival capacity and inactivation of the apoptotic pathways are the molecular mechanisms responsible for conventional drug resistance.

Tumor necrosis factor-related apoptosis-inducing ligand (TRAIL) is a member of the tumor necrosis factor cytokine family. TRAIL has been shown to induce apoptosis in cancer cells with minimal cytotoxicity toward non-transformed cells. TRAIL binds to several distinct receptors, death receptor (DR) 4/TRAIL receptor-1 (TRAIL-R1), DR5/TRAIL-R2, TRAIL-R3 and TRAIL-R4 (1). Both DR4 and DR5 contain an intracellular death domain that is essential for the induction of apoptosis following receptor ligation. In contrast, neither TRAIL-R3 nor TRAIL-R4 mediates apoptosis owing to a complete or partial lack of the intracellular death domain. These receptors are regarded as decoy receptors (DcRs) $(1,2)$. TRAIL activates the extrinsic apoptotic pathway. TRAIL binding to DR4/DR5 induces their oligomerization and conformational changes in the death domains, resulting in the formation of a death-inducing signaling complex and subsequent activation of caspase-8. In turn, activated caspase- 8 activates the effector caspase-3/6/7 that executes the apoptotic process $(3,4)$. The activation of caspase- 8 is also linked to the intrinsic (mitochondrial) apoptotic pathway. Activated caspase- 8 can cleave and activate the pro-apoptotic Bcl-2-family molecule Bid. In turn, truncated Bid activates other Bcl-2-family molecules, Bax and Bak, resulting in their oligomerization and the formation of megachannels in the outer mitochondrial membrane. The release of cytochrome $c$ through the Bax/Bak megachannels into the cytosol induces assembly of the apoptosome, representing the activation-platform for caspase-9. Caspase-9 also promotes the activation of caspase-3/6/7, thereby providing a positive loop for caspase activation (3). Some cancer cell types such as malignant melanoma cells are resistant to TRAIL despite their DR expres- 
sions on the cell surface (5). Moreover, TRAIL-responsive tumors acquire a resistant phenotype that renders TRAIL therapy ineffective. Therefore, overcoming TRAIL resistance is necessary for effective TRAIL therapy and drugs that potentiate TRAIL effectiveness are urgently required.

The garlic organosulfur compound, diallyl trisulfide (DATS) has anti-proliferative and/or pro-apoptotic effects in various cancer cell types including glioblastoma, melanoma, prostate cancer and colon cancer (6-8). In addition, DATS has been shown to potentiate TRAIL cytotoxicity toward human prostate cancer cells (9). The induction and/or amplification of apoptosis are associated with upregulation of multiple pro-apoptotic molecules, including DR4/DR5, Bax and Bak, downregulation of anti-apoptotic molecules such as Bcl-2 and Bcl-xL, and activation of caspases (8). The effects of DATS alone or in combination with TRAIL on the growth of TRAIL-resistant cells are poorly documented. Here we show that DATS can sensitize TRAIL-resistant human melanoma cells to DR-mediated apoptosis. Importantly, DATS shows its effects in a tumor-selective manner. Our data showed that DATS amplifies the cytotoxicity of death ligands by disrupting melanoma cell adaptation to endoplasmic reticulum (ER)-mediated apoptosis.

\section{Materials and methods}

Reagents. Reagents were obtained from the following manufacturers: soluble recombinant human TRAIL: Enzo Life Sciences (San Diego, CA, USA); DATS: Wako Pure Chemicals (Osaka, Japan); Thapsigargin (Tg): Sigma-Aldrich (St. Louis, MO, USA); z-VAD-fluoromethylketone (fmk) (VAD), z-DEVD-fmk (DEVD), z-IETD-fmk (IETD), and z-LETD-fmk (LETD): Calbiochem (La Jolla, CA, USA). z-LEVD-fmk (LEVD), z-ATAD-fmk (ATAD): BioVision (Mountain View, CA, USA); monoclonal antibodies against human DR4 and DR5: R\&D Systems (Minneapolis, MN, USA); polyclonal antibodies against X-box-binding protein-1 (XBP-1) and glucose-related protein 78 (GRP78): Santa Cruz Biotechnology (Santa Cruz, CA, USA). All other chemicals were of analytical grade. Reagents were dissolved in dimethylsulfoxide and diluted with high glucose-containing DMEM (Sigma-Aldrich) supplemented with $10 \%$ FBS (JRH Biosciences, Lenexa, KS, USA) to a final concentration of $<0.1 \%$ before use. Dimethylsulfoxide alone at a concentration of $0.1 \%$ (vehicle) had no effect throughout this study.

Cell culture. The human melanoma cell lines were obtained from Riken Bioresource Center Cell Bank (Tsukuba, Japan), Health Science Research Resource Bank (Osaka, Japan) or American Type Culture Collection (Manassas, VA, USA), and cultured in high glucose-containing DMEM supplemented with $10 \%$ FBS in a $5 \% \mathrm{CO}_{2}$-containing atmosphere. The cells were harvested by incubation in $0.25 \%$ trypsinEDTA medium (Gibco-Invitrogen, Carlsbad, CA, USA) for 5 min at $37^{\circ} \mathrm{C}$. Normal human epidermal melanocytes were obtained from Cascade Biologics (Portland, OR, USA) and cultured in DermaLife Basal Medium (Kurabo, Osaka, Japan) supplemented with DermaLife M LifeFactors (Kurabo) in a $5 \% \mathrm{CO}_{2}$-containing atmosphere. The cells were harvested by incubation in $0.25 \%$ trypsin-EDTA medium for $5 \mathrm{~min}$ at $37^{\circ} \mathrm{C}$.
Determination of cell death by fluorescence microscopy. Overall cell death was evaluated by fluorescence microscopy as previously described (10). Briefly, cells were placed on 8-chamber coverslips (Asahi Glass Co., Tokyo, Japan) and treated with the agents to be tested for $24 \mathrm{~h}$ at $37^{\circ} \mathrm{C}$ in a $5 \% \mathrm{CO}_{2}$-containing atmosphere. The cells were then stained with $4 \mu \mathrm{M}$ each of calcein-AM and ethidium bromide homodimer-1 (EthD-1) to label live and dead cells, respectively, using a commercially available kit (Live/Dead ${ }^{\circledR}$ Viability/Cytotoxicity Kit; Invitrogen) according to the manufacturer's instructions. Images were obtained with a fluorescence microscope (IX71 inverted microscope, Olympus, Tokyo, Japan) and analyzed using the LuminaVision software (Mitani Corporation, Fukui, Japan).

Determination of apoptotic cell death. Apoptotic cell death was quantitatively assessed by staining with PI and FITC-conjugated Annexin V as previously described (10) using a commercially available kit (Annexin V FITC Apoptosis Detection Kit I; BD Biosciences, San Jose, CA, USA). The stained cells were analyzed in a FACSCalibur (BD Biosciences) using the CellQuest software (BD Biosciences). Four cellular subpopulations were evaluated: vital cells (Annexin $\mathrm{V}^{-} / \mathrm{PI}^{-}$); early apoptotic cells (Annexin $\mathrm{V}^{+} / \mathrm{PI}^{-}$); late apoptotic cells (Annexin $\mathrm{V}^{+} / \mathrm{PI}^{+}$); and necrotic/damaged cells (Annexin $\mathrm{V}^{-} / \mathrm{PI}^{+}$). Annexin $\mathrm{V}^{+}$cells were considered to be apoptotic cells.

Determination of surface DR expression. The expressions of TRAIL-R1 and TRAIL-R2 on the cell surface were determined by flow cytometry. Briefly, cells $\left(5 \times 10^{5}\right.$ cells per $\left.20 \mu \mathrm{l}\right)$ in microtubes were incubated with anti-TRAIL-R1 and TRAIL-R2 antibodies (R\&D Systems, Minneapolis, MN, USA) or a subclass-matched IgG for $30 \mathrm{~min}$ at $4^{\circ} \mathrm{C}$. The cells were then centrifuged at $4^{\circ} \mathrm{C}$, resuspended in phosphate-buffered saline (PBS) and incubated with PE-conjugated goat $\mathrm{F}\left(\mathrm{ab}^{\prime}\right)_{2}$ anti-mouse IgG (R\&D Systems) for $30 \mathrm{~min}$ at $4^{\circ} \mathrm{C}$. Fluorescence was determined using the FL-2 channel of the FACSCalibur and analysed using the CellQuest software. Similar levels of fluorescence were observed in cells stained with the PE-conjugated subclassmatched IgG and unstained cells.

\section{Measurements of mitochondrial membrane potential $\left(\Delta \Psi_{m}\right)$ depolarization and caspase-3/7 activation. $\Delta \Psi_{\mathrm{m}}$ depolarization and caspase-3/7 activation were simultaneously measured as previously described (10). Briefly, cells plated in 24-well plates were treated with the agents to be tested in FBS/DMEM for $24 \mathrm{~h}$, stained with the dual sensor MitoCasp (Cell Technology Inc., Mountain View, CA, USA) and analyzed for their caspase-3/7 activity and $\Delta \Psi_{\mathrm{m}}$ in the FACSCalibur using the CellQuest soft- ware. Changes in $\Delta \Psi_{\mathrm{m}}$ were also measured using the lipophilic cation JC-1 as previously described (11).}

Measurement of caspase-12 activation. Activated caspase-12 in living cells was detected using the caspase-12 inhibitor ATAD conjugated to FITC as a marker as previously described (10). This compound binds to active caspase-12, but not to inactive caspase-12. Cells were stained with FITC-ATAD for $30 \mathrm{~min}$ at $37^{\circ} \mathrm{C}$ using a CaspGlow Fluorescein Caspase-12 staining kit (BioVision) according to the manufacturer's protocol. Fluorescence was determined using the FL-1 channel of the FACSCalibur and analyzed using the CellQuest software. 
Western blot analysis. Western blot analyses were carried out as previously described (10). Cells were treated with the agents to be tested for $24 \mathrm{~h}$ at $37^{\circ} \mathrm{C}$, washed and lysed with SDS-sample buffer. The whole cell lysates were subjected to SDS-PAGE and transferred to PVDF membranes (Nippon Millipore, Tokyo, Japan). After blocking the membranes with BlockAce (Dainippon Sumitomo Pharma, Osaka, Japan) at room temperature for $60 \mathrm{~min}$, GRP78, XBP-1, caspase-3 and caspase- 12 proteins on the membranes were detected using specific antibodies. Antibody-antigen complexes were detected with the ECL Prime Western Blotting Reagent (GE Healthcare Japan, Tokyo, Japan). To verify equal loading, the membranes were re-probed with an anti- $\beta$-actin or antiGAPDH antibody.

\section{Results}

DATS sensitizes human melanoma cells to TRAIL sparing normal cells. First, we examined the cytotoxic effects of TRAIL and/or DATS (chemical structure is shown in Fig. 1A) toward melanoma cells. The human melanoma cell lines A375, A2058 and SK-MEL-2 were treated with DATS and TRAIL alone or in combination for $24 \mathrm{~h}$, stained with calcein-AM and EthD-1 and observed under a fluorescence microscope (Fig. 1B-D). Live cells were stained green with calcein-AM, while dead cells with compromised cell membranes were stained red with EthD-1. These cells were highly resistant to $100 \mathrm{ng} / \mathrm{ml}$ TRAIL and DATS had only a modest cytotoxic effect. However, the combined use of TRAIL and DATS caused considerable cell death. On the contrary, DATS and TRAIL alone or in combination induced minimal cell death in the primary melanocytes (Fig. 1E). These data show that DATS sensitizes human melanoma cells to TRAIL-induced apoptosis while sparing normal cells. To confirm the tumor-selective actions of DATS, we evaluated apoptosis by flow cytometry using Annexin V/PI staining. TRAIL treatment resulted in apoptotic cell death in a dose- and time-dependent manner. However, the effect was only modest (maximum of $30 \%$ increase in Annexin $\mathrm{V}^{+}$cells), even after treatment with $100 \mathrm{ng} / \mathrm{ml}$ TRAIL for $72 \mathrm{~h}$ (Fig. 2A). Although $100 \mu \mathrm{M}$ DATS alone caused little apoptosis, it substantially potentiated TRAIL-induced apoptosis for $24 \mathrm{~h}$ (Fig. 2B). On the contrary, DATS and TRAIL alone or in combination caused minimal apoptosis in melanocytes despite their substantial surface expression of DR4 and DR5 (Fig. 2B and C). The amplification of TRAIL-induced apoptosis was initially observed after $24 \mathrm{~h}$ of treatment (Fig. 2B) and a more pronounced effect was observed after $72 \mathrm{~h}$ of treatment (Fig. 2D). Consequently, strong apoptosis (maximum of 70\%) was induced by the combined use of $25 \mathrm{ng} / \mathrm{ml}$ TRAIL and DATS (Fig. 2D). These data further indicate that DATS sensitizes human melanoma cells to TRAIL while sparing normal cells. Therefore, we subsequently investigated the mechanisms of the sensitization using A375 cells as a model cell system.

DATS potentiates apoptotic signaling through DR4/DR5. TRAIL binds to not only DR4 and DR5, but also DcRs, triggering cross-linking of these receptors into homo- and/or heterotrimers $(12,13)$. In contrast, agonistic monoclonal antibodies against DR4 or DR5 trigger the formation of multimeric complexes containing only one specific DR, which
A

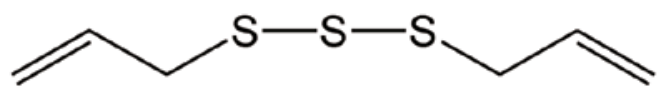

B

A375 cells

C A2058 cells

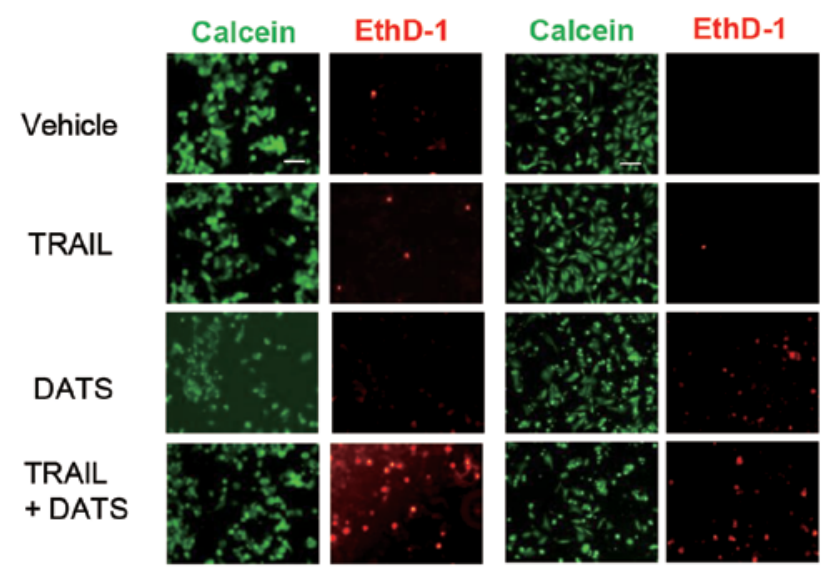

D

SK-MEL-2 cells

E Melanocytes

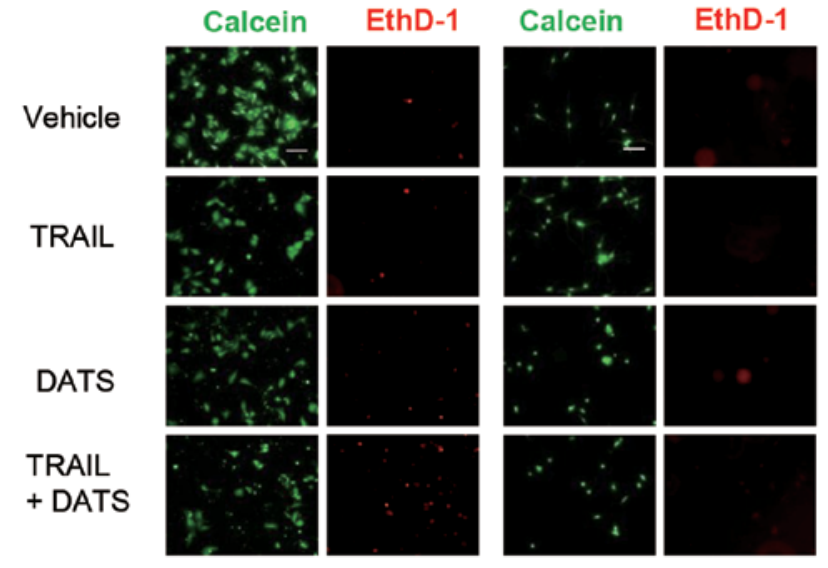

Figure 1. DATS potentiates TRAIL cytotoxicity toward different human melanoma cell lines, but not melanocytes. (A) Chemical structure of DATS (B) A375 cells, (C) A2058 cells, (D) SK-MEL-2 cells and (E) melanocytes were plated on 8 -chamber coverslips and treated with $25 \mathrm{ng} / \mathrm{ml}$ TRAIL and $100 \mu \mathrm{M}$ DATS alone or in combination for $24 \mathrm{~h}$. After removal of the medium, the cells were stained with calcein-AM and EthD-1 to label vital cells (green) and dead cells with compromised cell membranes (red), respectively. Images were obtained using a fluorescence microscope. The data are representative of 3 independent experiments. Scale bar, $100 \mu \mathrm{m}$.

consequently enables them to bypass signaling through the DcRs (14,15). Addition of an anti-DR5 antibody (anti-DR5) at concentrations of $\geq 1 \mu \mathrm{g} / \mathrm{ml}$ induced substantial apoptosis in a dose-dependent manner after $24 \mathrm{~h}$ of treatment, whereas an anti-DR4 antibody (anti-DR4) at the same concentra- 
A

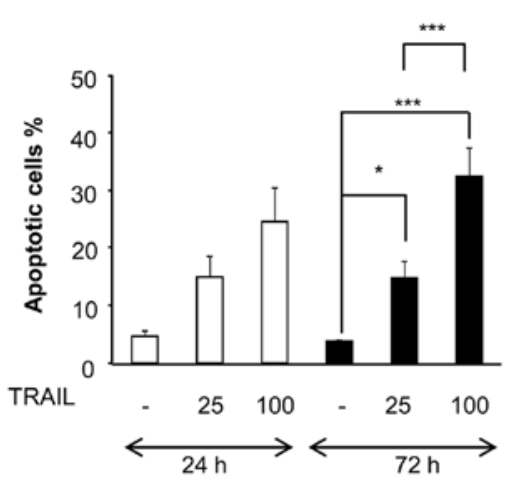

C
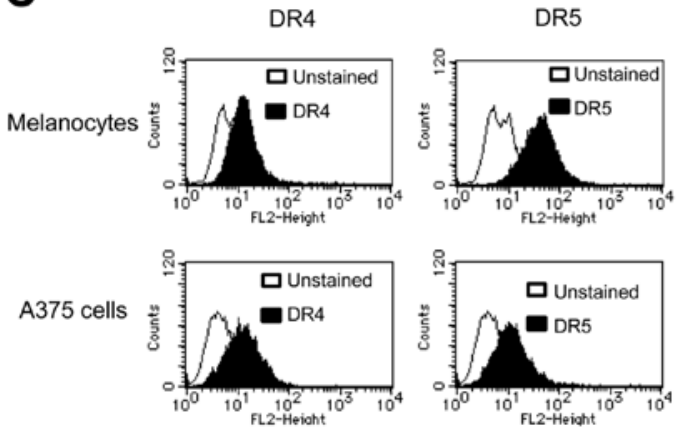

B

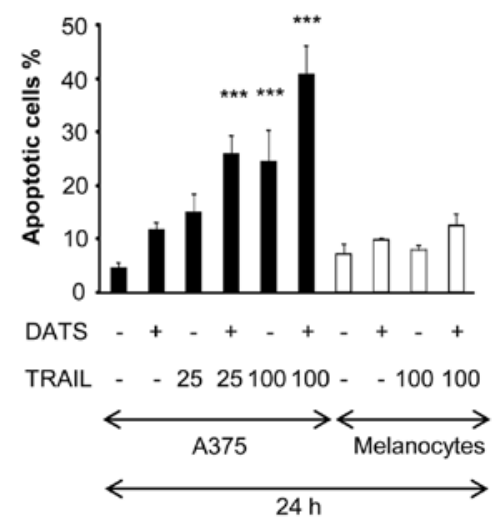

D

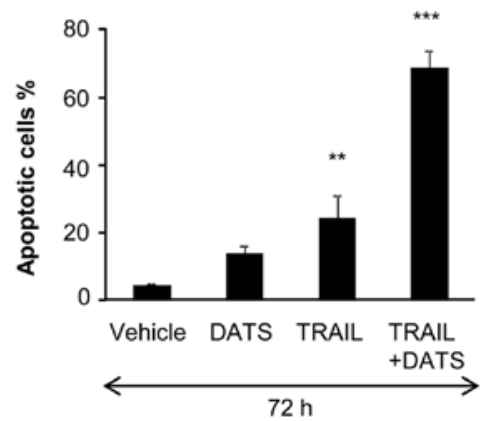

Figure 2. DATS sensitizes melanoma cells, but not melanocytes, to TRAIL-induced apoptosis. (A and B) A375 cells or melanocytes were plated in 24-well plates, treated with TRAIL at the indicated concentrations for the indicated time in the (A) absence or (B) presence of $100 \mu \mathrm{M}$ DATS for $24 \mathrm{~h}$ and analyzed for their apoptosis by Annexin V/PI staining and flow cytometry. Annexin $\mathrm{V}^{+}$cells were considered to be apoptotic cells. The data represent means $\pm \mathrm{SE}$ from 3 to 9 experiments. ${ }^{*} \mathrm{P}<0.05 ;{ }^{* *} \mathrm{P}<0.01 ;{ }^{* * *} \mathrm{P}<0.001$. (C) Melanocytes were analyzed for their expression of DR4 and DR5 by indirect immunofluorescence and flow cytometry. (D) A375 cells were treated with $25 \mathrm{ng} / \mathrm{ml}$ TRAIL and $100 \mu \mathrm{M}$ DATS alone or in combination for $72 \mathrm{~h}$ and apoptotic cell death was measured as described above. The data represent means \pm SE from 8 experiments.

A

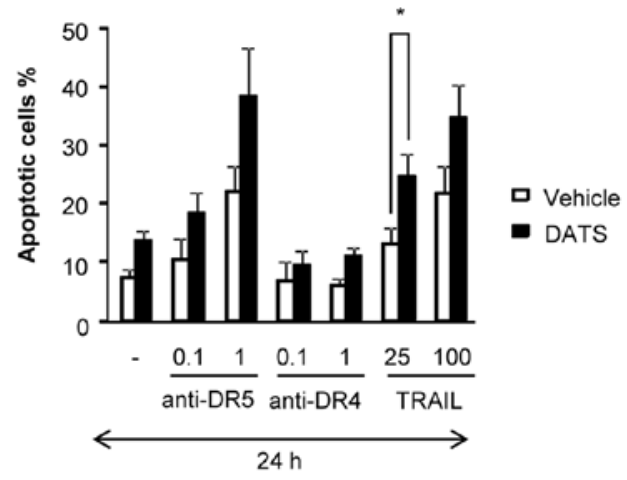

B

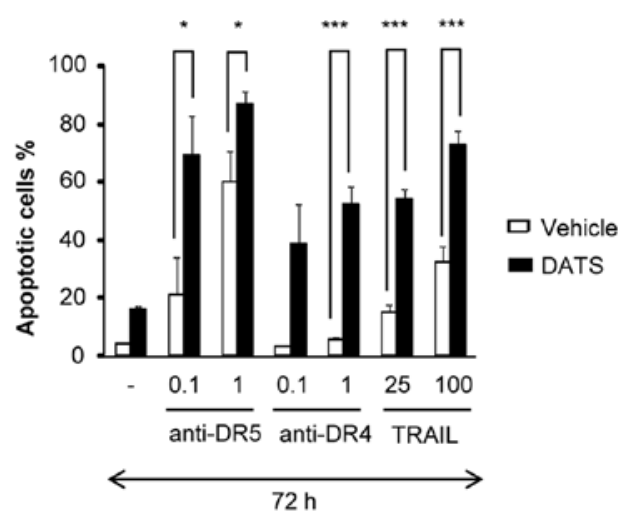

Figure 3. DATS potentiates apoptosis through DR4/DR5. (A and B) A375 cells were treated with TRAIL ( $25 \mathrm{or} 100 \mathrm{ng} / \mathrm{ml})$, anti-DR4 $(0.1 \mathrm{or} 1 \mu \mathrm{g} / \mathrm{ml})$, and anti-DR5 (0.1 or $1 \mu \mathrm{g} / \mathrm{ml}$ ) and $100 \mu \mathrm{M}$ DATS alone or in combination for (A) 24 or (B) $72 \mathrm{~h}$, and apoptotic cell death was measured by Annexin V/PI staining and flow cytometry. Annexin $\mathrm{V}^{+}$cells were considered to be apoptotic cells. The data represent means \pm SE from 3 to 10 experiments. ${ }^{*} \mathrm{P}<0.05 ;{ }^{* * *} \mathrm{P}<0.001$.

tions caused minimal apoptosis, and DATS amplified the effect of anti-DR5, but not anti-DR4 (Fig. 3A). In contrast, isotype-matched Ig controls for anti-DR5 and anti-DR4 had no such effects (data not shown). On the other hand, considerable apoptosis $(20 \%)$ was observed after $72 \mathrm{~h}$ of treatment with DATS and anti-DR5 or anti-DR4 (Fig. 3B). These data 
A

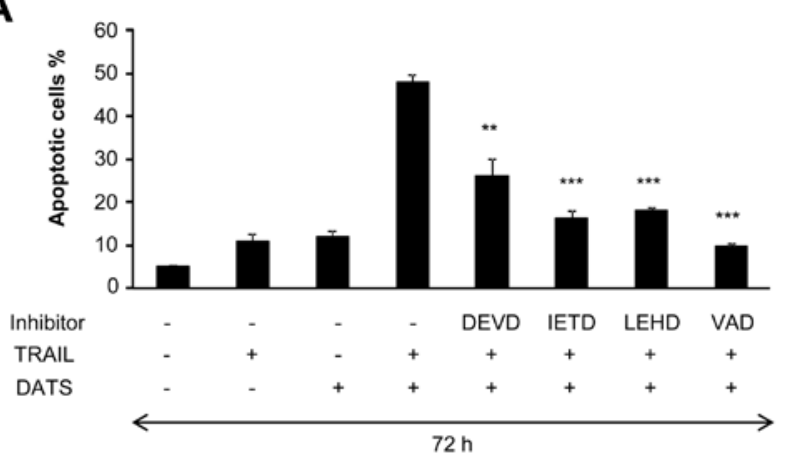

B

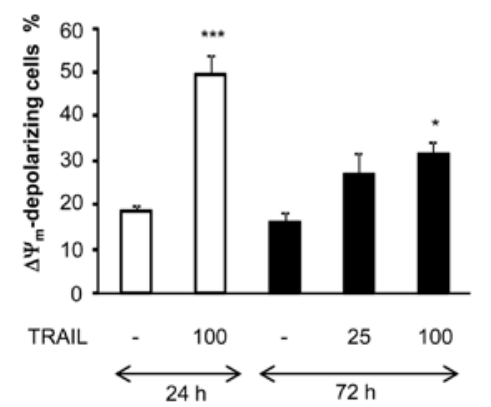

D

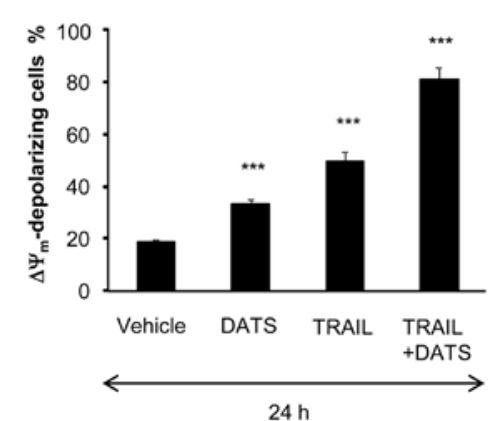

C

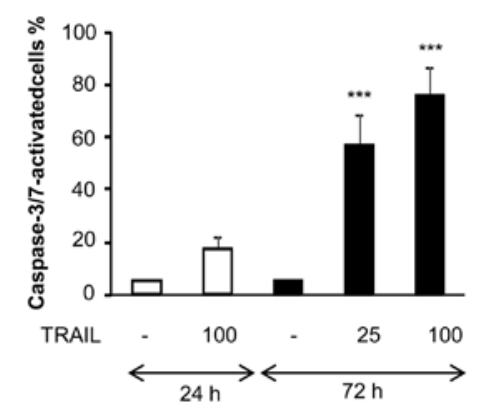

E

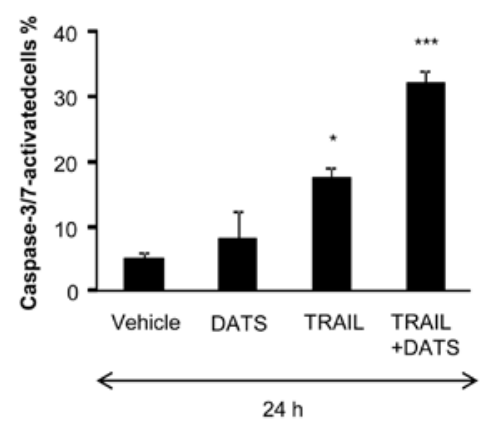

F

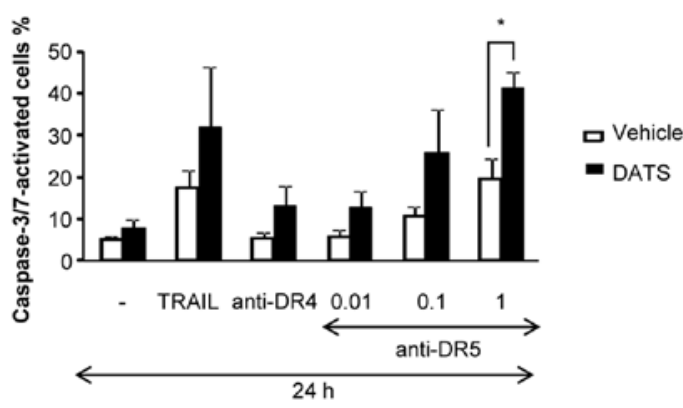

G

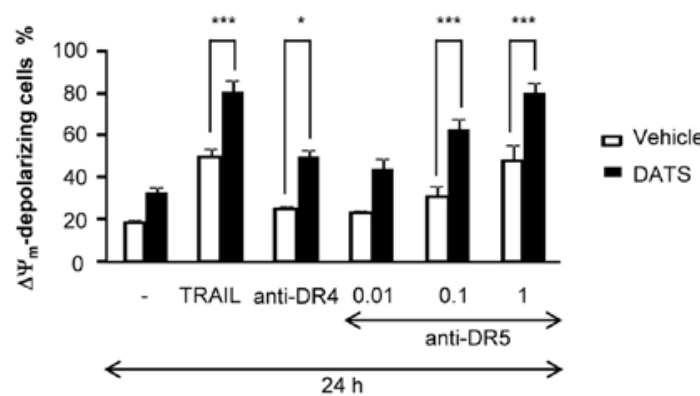

H

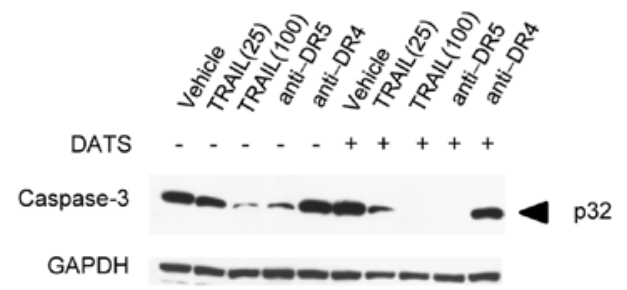

Figure 4. Intrinsic apoptotic pathway is not sufficient for sensitization by DATS. (A) A375 cells were treated with 25 ng/ml TRAIL and $100 \mu$ M DATS alone or in combination in the presence or absence of $10 \mu \mathrm{M}$ each of z-DEVD-fmk (DEVD), z-IETD-fmk (IETD), z-LEHD-fmk (LEHD) or z-VAD-fmk (VAD) for $72 \mathrm{~h}$. Apoptotic cells were measured by Annexin V/PI staining and flow cytometry. Annexin $\mathrm{V}^{+}$cells were considered to be apoptotic cells. The data represent means \pm SE from 3 to 8 experiments. ${ }^{* *} \mathrm{P}<0.01 ;{ }^{* * *} \mathrm{P}<0.001$. (B and $\mathrm{C}$ ) A375 cells were treated with TRAIL at the indicated concentrations for the indicated time, and (B) $\Delta \Psi_{\mathrm{m}}$ depolarization and (C) caspase-3/7 activation were determined by flow cytometry. The data represent means \pm SE from 3 to 6 experiments. ${ }^{*} \mathrm{P}<0.05$; ${ }^{* * *} \mathrm{P}<0.001$. (D and E) A375 cells were treated with $100 \mathrm{ng} / \mathrm{ml}$ TRAIL and $100 \mu \mu \mathrm{M}$ DATS alone or in combination for $24 \mathrm{~h}$, and (D) $\Delta \Psi_{\mathrm{m}}$ depolarization and (E) caspase-3/7 activation were determined by flow cytometry. The data present means \pm SE from 3 experiments. ${ }^{*} \mathrm{P}<0.05$; ${ }^{* * *} \mathrm{P}<0.001$. (F and $\left.\mathrm{G}\right) \mathrm{A} 375$ cells were treated with TRAIL $(100 \mathrm{ng} / \mathrm{ml})$, anti-DR4 $(1 \mu \mathrm{g} / \mathrm{ml})$ or anti-DR5 $(0.01,0.1$ or $1 \mu \mathrm{g} / \mathrm{ml})$ alone or in combination with $100 \mu \mathrm{M}$ DATS for $24 \mathrm{~h}$, and analyzed for their $(\mathrm{F})$ caspase-3/7 activation and $(\mathrm{G}) \Delta \Psi_{\mathrm{m}}$ depolarization. The data represent means $\pm \mathrm{SE}$ from 3 experiments. ${ }^{*} \mathrm{P}<0.05$; ${ }^{* * * *} \mathrm{P}<0.001$. $(\mathrm{H}) \mathrm{A} 375$ cells were treated with TRAIL ( 25 or $100 \mathrm{ng} / \mathrm{ml})$, anti-DR4 $(1 \mu \mathrm{g} / \mathrm{ml})$ or anti-DR5 $(1 \mu \mathrm{g} / \mathrm{ml})$ alone or in combination with $100 \mu \mathrm{M}$ DATS for $24 \mathrm{~h}$, and analyzed for their contents of caspase-3 (p32) by western blot analyses. To verify equal loading, the membranes were reprobed with an anti-GAPDH antibody. The data are representative of 4 independent experiments.

suggest that DATS potentiates the apoptotic signals through DR4/DR5 rather than putative survival signals through DcRs.

The mitochondrial apoptotic pathway is not sufficient for the sensitization. We examined the role of caspases in the sensitization by DATS. As shown in Fig. 4A, the general caspase inhibitor VAD completely abolished the sensitization, indicating that it was caspase-dependent. The caspase-8-specific inhibitor IETD and caspase-9-specific inhibitor LEHD also strongly reduced the sensitization (maximum of $75 \%$ inhibition), whereas the caspase-3/7-specific inhibitor DEVD was less effective (maximum of 50\%). To obtain further evidence for the role of the intrinsic death pathway, we measured $\Delta \Psi_{\mathrm{m}}$ and caspase-3/7 activation. Strikingly, despite its poor apoptosis-inducing effect, treatment with TRAIL at concentrations of $\geq 25 \mathrm{ng} / \mathrm{ml}$ for $72 \mathrm{~h}$ induced substantial $\Delta \Psi_{\mathrm{m}}$ collapse and caspase-3/7 activation in a dose- and time-dependent manner (Fig. 4B and C). The induction of $\Delta \Psi_{\mathrm{m}}$ collapse was confirmed using another $\Delta \Psi_{\mathrm{m}}$-sensitive dye JC-1 (data not shown). On the other hand, at a higher concentration, TRAIL caused substantial $\Delta \Psi_{\mathrm{m}}$ collapse and caspase-3/7 activation within $24 \mathrm{~h}$. DATS alone caused robust $\Delta \Psi_{\mathrm{m}}$ collapse, but not caspase-3/7 activation. However, 

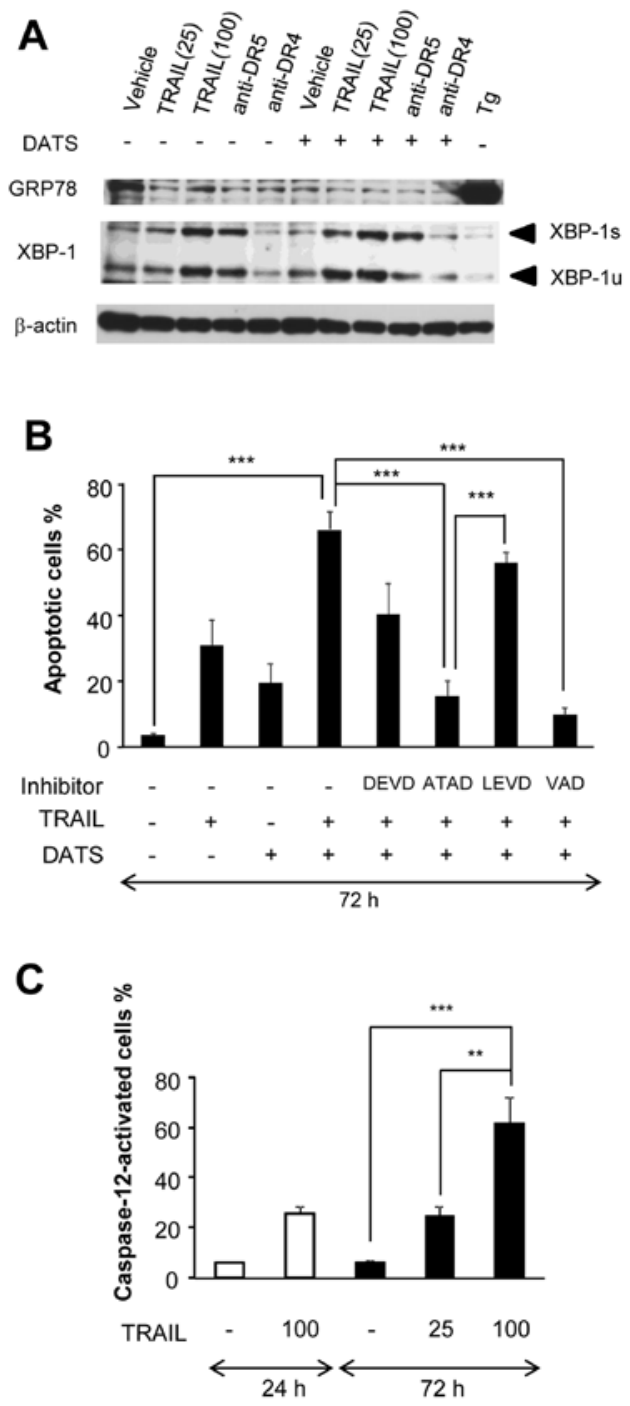

D
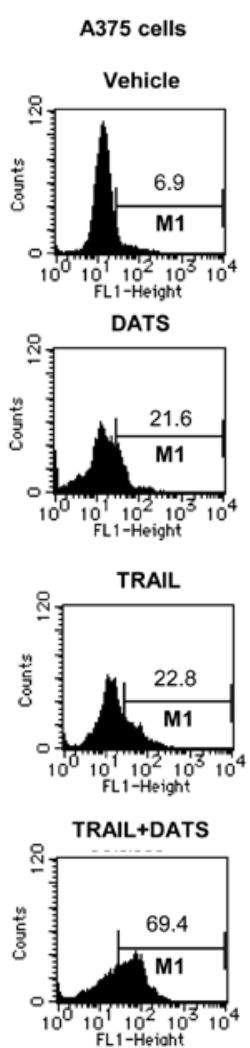
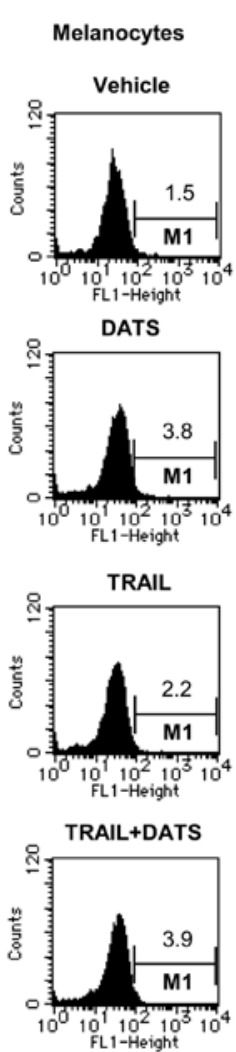

$\mathbf{E}$
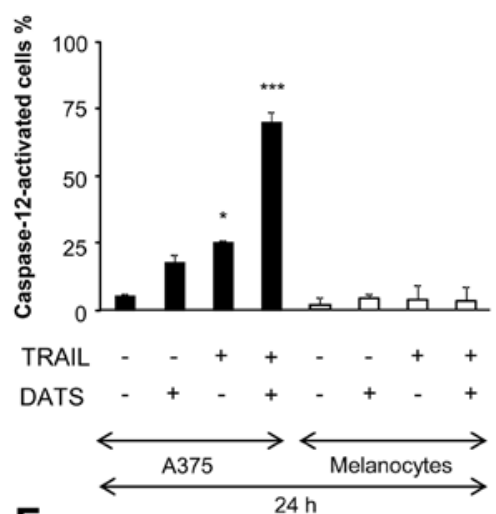

$\mathbf{F}$

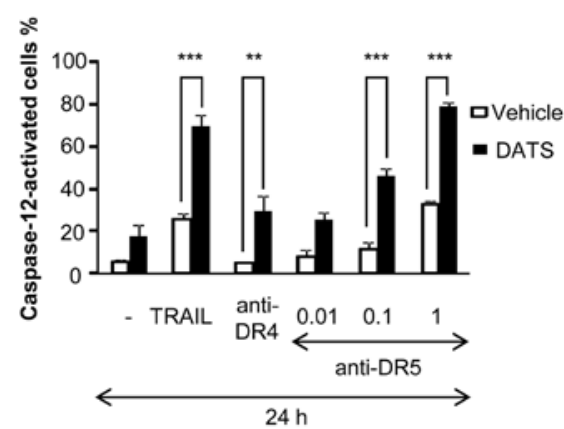

G

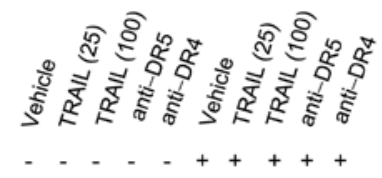

Caspase-12 $=-\cdots-\cdots,-\cdots \mathrm{p} 55$

GAPDH

Figure 5. DATS enhances TRAIL-induced ER stress and caspase-12 activation. (A) A375 cells were treated with TRAIL ( $25 \mathrm{or} 100 \mathrm{ng} / \mathrm{ml})$, anti-DR4 (1 $\mu \mathrm{g} / \mathrm{ml})$ or anti-DR5 $(1 \mu \mathrm{g} / \mathrm{ml})$ alone or in combination with $100 \mu \mathrm{M}$ DATS for $24 \mathrm{~h}$. Thapsigargin $(\mathrm{Tg} ; 1 \mu \mathrm{M})$ served as a positive control for ER stress response. The cells were analyzed for their contents of GRP78 and XBP-1 proteins by western blot analyses. To verify equal loading, the blots were reprobed with an anti- $\beta$-actin antibody. The data are representative of 3 independent experiments. (B) A 375 cells were treated with $25 \mathrm{ng} / \mathrm{ml}$ TRAIL and $100 \mu$ M DATS alone or in combination in the presence or absence of $10 \mu \mathrm{M}$ each of z-DEVD-fmk (DEVD), z-ATAD-fmk (ATAD), z-LEVD-fmk (LEVD) or z-VAD-fmk (VAD) for $72 \mathrm{~h}$. Apoptotic cells were measured by Annexin V/PI staining and flow cytometry. Annexin $\mathrm{V}^{+}$cells were considered to be apoptotic cells. The data represent means \pm SE from 3 experiments. ${ }^{* * *} \mathrm{P}<0.001$. (C) A375 cells were treated with TRAIL ( 25 or $100 \mathrm{ng} / \mathrm{ml}$ ) for $24 \mathrm{or} 72 \mathrm{~h}$, and caspase-12 activity was measured using FITC-ATAD-fmk. The data are shown as percentages of caspase-12-activated cells and represent means \pm SE from 3 experiments. ${ }^{* *} \mathrm{P}<0.01{ }^{* * * *} \mathrm{P}<0.001$. (D and $\left.\mathrm{E}\right) \mathrm{A} 375$ cells and melanocytes were treated with $100 \mathrm{ng} / \mathrm{ml}$ TRAIL and $100 \mu \mathrm{M}$ DATS alone or in combination for $24 \mathrm{~h}$, and caspase-12 activity was measured as described above. A typical histogram is shown in (D). The data shown in (E) represent means $\pm \mathrm{SE}$ from 3 experiments. ${ }^{*} \mathrm{P}<0.05 ;{ }^{* * * *} \mathrm{P}<0.001$. (F and $\left.\mathrm{G}\right) \mathrm{A} 375$ cells were treated with TRAIL ( 25 or $100 \mathrm{ng} / \mathrm{ml})$, anti-DR4 $(1 \mu \mathrm{g} / \mathrm{ml})$ or anti-DR5 $(1 \mu \mathrm{g} / \mathrm{ml})$ alone or in combination with $100 \mu \mathrm{M}$ DATS for $24 \mathrm{~h}$. The cells were (F) measured for their caspase-12 activity as described above or (G) analyzed for their contents of full-length caspase-12 (p55) by western blot analysis. To verify equal loading, the blots were reprobed with the anti-GAPDH antibody.

in parallel with apoptosis, higher degrees of $\Delta \Psi_{\mathrm{m}}$ depolarization and caspase-3/7 activation were observed in cells treated with TRAIL and DATS in combination than in cells treated with either agent alone (Fig. 4D and E). DATS also enhanced both $\Delta \Psi_{\mathrm{m}}$ depolarization and caspase-3/7 activation induced by anti-DR5 and by anti-DR4 to lesser extent (Fig. 4F and G). The activation of caspase-3/7 was also demonstrated by western blot analysis. TRAIL, but not DATS, alone induced substantial processing of caspase-3. In parallel with apoptosis, anti-DR5, but not anti-DR4, induced robust caspase-3 processing after $24 \mathrm{~h}$ of treatment and DATS enhanced the effect (Fig. 4H). Collectively, these data suggest that the intrinsic death pathway is involved in the amplification of apoptosis, but is not sufficient for full sensitization.

DATS enhances DR-mediated ER stress and caspase-12 activation. A growing body of evidence suggests that the $\mathrm{ER}$ is a key player in the regulation of apoptosis, and that ER stress is the major cause of a variety of pro-apoptotic effects (16-18). To elucidate the role of ER stress in the sensitization, we first examined whether TRAIL caused the unfolded protein response (UPR), a cellular response to ER stress. After treatment with TRAIL for $24 \mathrm{~h}$, the cells were analyzed for their expression of two UPR proteins, GRP78 and XBP1 by western blot analysis. 
Since $\mathrm{Tg}$, an inhibitor of sarco/endoplasmic reticulum $\mathrm{Ca}^{2+}$ ATPase was shown to be a potent inducer of ER stress in human melanoma cells (19), it served as a positive control. Tg treatment caused a considerable increase in GRP78 expression, whereas TRAIL treatment did not (Fig. 5A). On the other hand, TRAIL dose-dependently increased the expression of XBP-1, although the degrees varied considerably in different experiments. For XBP-1, the active spliced form (XBP-1s) and inactive unspliced form (XBP-1u) were increased by 1.8-5.6-fold and 2.7-7.6-fold, respectively. Anti-DR5 showed similar effects (XBP-1u and XBP-1s were increased by 3.8- and 5.6-fold, respectively), whereas anti-DR4 did not (Fig. 5A). There was a tendency that the combined use of DATS and TRAIL decreased the expression of GRP78. On the other hand, DATS alone had minimal effects on the expression of both forms of XBP-1, whereas it enhanced the effects of TRAIL on their expression. In parallel with apoptosis, the enhancement was observed more pronouncedly in cells stimulated with $25 \mathrm{ng} / \mathrm{ml}$ TRAIL than in cells stimulated with $100 \mathrm{ng} / \mathrm{ml}$ TRAIL. Similarly, anti-DR5 induced substantial increase in the expressions of XBP-1s and XBP-1u, whereas anti-DR4 did not (Fig. 5A). To elucidate the possible roles of ER-associated caspase-12 and caspase- 4 in the sensitization, we examined the effects of their specific inhibitors $(10 \mu \mathrm{M})$. The caspase-12-specific inhibitor ATAD almost completely abrogated the sensitization, whereas the caspase-4-specific inhibitor LEVD had a minimal effect (Fig. 5B). Similar results were obtained with the two inhibitors at $30 \mu \mathrm{M}$ (data not shown). These data suggest that caspase-12 is important for the sensitization in our systems. To further explore the role of caspase-12, we examined whether TRAIL and/or DATS affected the activation of the enzyme. The functional activation of caspase-12 was assessed by measuring the conversion of a cell-permeable substrate, FITC-ATAD-fmk. The results revealed that TRAIL induced robust caspase-12 activation in a dose- and time-dependent manner (Fig. 5C). In addition, DATS significantly enhanced the effects of TRAIL, anti-DR5 and anti-DR4 with varying degrees (Fig. 5D-F). The enhancement of TRAIL-induced caspase-12 activation was also shown by its processing, providing further molecular evidence for the activation of the enzyme. In parallel with apoptosis, anti-DR5, but not anti-DR4, induced robust caspase-12 processing and synergized with DATS to induce this effect (Fig. 5G). By contrast, DATS and TRAIL alone or in combination caused minimal caspase-12 activation in melanocytes (Fig. 5D, E). These data show that DATS also enhances the ER-mediated apoptotic pathway involving caspase-12 activation in a tumor-selective manner.

\section{Discussion}

The data obtained in this study clearly demonstrated that DATS sensitizes human melanoma cells to DR-mediated apoptosis. DATS and TRAIL alone had little cytotoxicity toward these cells. Nevertheless, the combined use of DATS and TRAIL caused considerable apoptotic cell death. TRAIL binds to not only DR4 and DR5 but also DcRs, triggering cross-linking of these receptors into homo- and/or heterotrimers $(12,13)$. Furthermore, DcRs have been shown to trigger putative survival signaling pathways, such as Bcl-2 and Akt thereby compromising the DR-mediated death signals (20). DcR1 and DcR2 have been shown to inhibit TRAIL-mediated DR5-death-inducing signaling complex formation (21). Therefore, there was the possibility that the sensitization was caused by downregulation of these survival events. However, our data showed that DATS also sensitized melanoma cells to apoptosis induced by agonistic monoclonal antibodies against DR4 or DR5. Consequently, the amplification of apoptosis may be caused by upregulation of death signaling rather than downregulation of survival signaling. It is noteworthy that DATS used alone or in combination with TRAIL had minimal cytotoxic effects toward the primary melanocytes, indicating that DATS acts in a tumor-selective manner. These data are similar to those in previous reports showing that garic organosulfur compounds such as diallyl disulfide induce apoptosis in cancer cells, but have no effect on normal cells (8).

Garic organosulfur compounds including DATS have been shown to induce apoptosis in various cancer cell types including glioblastoma (6), prostate (7), colon (22) and breast cancer cells (23). The intrinsic (mitochondrial) death pathway has also been shown to play important roles in the cytotoxicity of DATS $(6,7)$ and DATS-mediated amplification of TRAIL-induced apoptosis in prostate cancer cells (9). Consistent with these reports, our findings indicated that DATS-mediated amplification of TRAIL-induced apoptosis in melanoma cells was caspase-dependent and associated with increased $\Delta \Psi_{\mathrm{m}}$ collapse and effector caspase-3/7 activation. However, both caspase-9and caspase-3/7-specific inhibitors only partially inhibited the effect of DATS. In addition, $\Delta \Psi_{\mathrm{m}}$ collapse and caspase-3/7 activation did not necessarily parallel with apoptosis. It is noted that melanoma cells are relatively resistant to DATS compared with other cancer cells, such as prostate and colon cancer cells. In these cells, DATS at concentrations of 10-100 $\mu \mathrm{M}$ caused considerable apoptosis within $24 \mathrm{~h}$, achieving complete cell killing at around $50 \mu \mathrm{M}(6,7)$. On the other hand, all the melanoma cell lines tested in this study underwent only modest apoptosis $(<20 \%)$ after treatment with $100 \mu \mathrm{M}$ DATS for $72 \mathrm{~h}$. Collectively, it is possible to speculate that the intrinsic apoptotic pathway is sufficient to induce or amplify substantial apoptosis in prostate and colon cancer cells, but not in melanoma cells. Induction of apoptosis by the intrinsic pathway appears to be the major mechanism of conventional chemotherapy, and a critical target in cancer therapy. However, this pathway does not seem to be a suitable target in the treatment of TRAIL-resistant cancers including malignant melanoma, since chemotherapy is ineffective (24). Our data are consistent with the clinical observations and suggest that another caspase-dependent pathway is also required for effective induction of apoptosis in melanoma cells.

TRAIL induced robust ER stress responses such as activation of XBP-1 and caspase-12, and that DATS potentiated these effects in parallel with apoptosis. Caspase-12 is localized to the ER membrane, specifically activated by ER stress, and crucial for ER-mediated apoptosis $(25,26)$. Therefore, these findings suggest a role for caspase-12 and ER-mediated apoptosis in the sensitization by DATS. In fact, a specific inhibitor of caspase12 , but not caspase-4, strongly abolished the amplification of apoptosis. It is noteworthy that adaptation to ER stress is considered to be important for malignancy and resistance to therapy of cancer cells, including melanoma cells (27-29). ER stress is characterized by the accumulation of misfolded proteins and is caused by a variety of cellular conditions such as glucose deprivation, hypoxia, disturbance of calcium homeostasis, and excess reactive oxidant species production. ER stress activates 
the UPR, which downregulates protein synthesis, upregulates chaperone proteins, and increase protein degradation. If UPR activation is not able to relieve the ER stress, the cells undergo apoptosis $(17,18)$. Therefore, disruption of the adaptation may cause ER-mediated apoptosis in melanoma cells. The ER can initiate cell death through a pathway that is independent of mitochondria and DRs (31-32). An increasing body of evidence suggests that ER-mediated apoptosis and/or caspase-12 play crucial roles in the apoptosis induced by divergent chemicals in a variety of cancer cell types, including melanoma and skin cancer cells (30,33-35). Recently, we have shown the pro-apoptotic role of plasma membrane depolarization in TRAIL-induced apoptosis in melanoma cells. Consequently, membrane-depolarizing drugs such as ATP-sensitive $\mathrm{K}^{+}$ channel inhibitors sensitize melanoma cells to TRAIL-induced apoptosis (10). Interestingly, melanocytes are insensitive to TRAIL-induced depolarization and apoptosis as well as sensitization by membrane-depolarizing drugs, thereby indicating that the depolarization-mediated sensitization is tumor-specific. Similar to DATS, the intrinsic death pathway is insufficient for the depolarization-mediated sensitization. It is noteworthy that DATS and membrane-depolarizing drugs commonly remarkably potentiate TRAIL-induced ER stress response, including activation of XBP-1, since XBP-1 plays a pro-apoptotic role in melanoma cells by upregulating DR5 expression (29). Collectively, these findings expand the emerging view that adaptation of ER stress is a mechanism of melanoma resistance to therapy and suggest that disruption of the adaptation is a powerful way to amplify TRAIL effectiveness toward resistant cancer cells without impairing its tumor-selectivity.

In conclusion, we have demonstrated for the first time that DATS sensitizes human melanoma cells to DR-mediated cell death by disrupting their adaptation to ER-mediated apoptosis. The findings raise the possibility that DATS may be combined with death ligands to treat TRAIL-resistant cancers.

\section{Acknowledgements}

The authors thank Dr H. Nagase for providing the human melanoma cell lines. This study was supported in part by a Grant-in-Aid from the Ministry of Education, Culture, Sports, Science and Technology (KAKENHI 23591631; to YS) and a Grant-in-Aid from Nihon University (to YS).

\section{References}

1. LeBlanc HN and Ashkenazi A: Apo2L/TRAIL and its death and decoy receptors. Cell Death Differ 10: 66-75, 2003.

2. Johnstone RW, Frew AJ and Smyth MJ: The TRAIL apoptotic pathway in cancer onset, progression and therapy. Nat Rev Cancer 8: 782-798, 2008.

3. Wang S: The promise of cancer therapeutics targeting the TNF-related apoptosis-inducing ligand and TRAIL receptor pathway. Oncogene 27: 6207-6215, 2008.

4. Sayers TJ: Targeting the extrinsic apoptosis signaling pathway for cancer therapy. Cancer Immunol Immunother 60: 1173-1180, 2011.

5. Dyer MJ, MacFarlane M and Cohen GM: Barriers to effective TRAIL-targeted therapy of malignancy. J Clin Oncol 25: 4505-4506, 2007.

6. Das A, Banik NL and Ray SK: Garlic compounds generate reactive oxygen species leading to activation of stress kinases and cysteine proteases for apoptosis in human glioblastoma T98G and U87MG cells. Cancer 110: 1083-1095, 2007.
7. Kim YA, Xiao D, Xiao H, et al: Mitochondria-mediated apoptosis by diallyl trisulfide in human prostate cancer cells is associated with generation of reactive oxygen species and regulated by Bax/Bak. Mol Cancer Ther 6: 1599-1609, 2007.

8. Powlny AA and Singh SV: Multitargeted prevention and therapy of cancer by diallyl trisulfide and related Allium vegetablederived organosulfur compounds. Cancer Lett 269: 305-314, 2008.

9. Shankar S, Chen Q, Ganapathy S, Singh KP and Srivastava RK: Diallyl trisulfide increases the effectiveness of TRAIL and inhibits prostate cancer growth in an orthotropic model: molecular mechanisms. Mol Cancer Ther 7: 2328-2338, 2008.

10. Suzuki Y, Inoue T, Murai M, Suzuki-Karasaki M, Ochiai T and Ra C: Depolarization potentiates TRAIL-induced apoptosis in human melanoma cells: role for ATP-sensitive $\mathrm{K}^{+}$channels and endoplasmic reticulum stress. Int J Oncol 41: 465-475, 2012

11. Suzuki Y, Yoshimaru T, Inoue T and Ra C: Mitochondrial $\mathrm{Ca}^{2+}$ flux is a critical determinant of the $\mathrm{Ca}^{2+}$ dependence of mast cell degranulation. J Leukoc Biol 79: 508-518, 2006.

12. Griffith TS, Rauch CT, Smolak PJ, et al: Functional analysis of TRAIL receptors using monoclonal antibodies. J Immunol 162: 2597-2605, 1999.

13. Kischkel FC, Lawrence DA, Tinel A, et al: Death receptor recruitment of endogenous caspase-10 and apoptosis initiation in the absence of caspase-8. J Biol Chem 276: 46639-46646, 2001.

14. Pukac L, Kanakaraj P, Humphreys R, et al: HGS-ETR1, a fully human TRAIL-receptor 1 monoclonal antibody, induces cell death in multiple tumour types in vitro and in vivo. Br J Cancer 92: 1430-1441, 2005

15. Georgakis GV, Li Y, Humphreys R, et al: Activity of selective fully human agonistic antibodies to the TRAIL death receptors TRAIL-R1 and TRAIL-R2 in primary and cultured lymphoma cells: induction of apoptosis and enhancement of doxorubicinand bortezomib-induced cell death. Br J Haematol 130: 501-510, 2005.

16. Boyce $M$ and Yuan J: Cellular response to endoplasmic reticulum stress: a matter of life or death. Cell Death Differ 13: 363-373, 2006

17. Breckenridge DG, Germain M, Mathai JP, et al: Regulation of apoptosis by endoplasmic reticulum pathways. Oncogene 22: 8608-8618, 2003.

18. Groenendyk $\mathrm{J}$ and Michalak M: Endoplasmic reticulum quality control and apoptosis. Acta Biochim Pol 52: 381-395, 2005.

19. Chen LH, Jiang CC, Kiejda KA, et al: Thapsigargin sensitizes human melanoma cells to TRAIL-induced apoptosis by up-regulation of TRAIL-R2 through the unfolded protein response. Carcinogenesis 28: 2328-2336, 2007.

20. Mahalingam D, Szegezdi E, Keane M, de Jong S and Samali A: TRAIL receptor signalling and modulation: are we on the right TRAIL? Cancer Treat Rev 35: 280-288, 2009.

21. Merino D, Lalaoui N, Morizot A, Schneider P, Solary E and Micheau O: Differential inhibition of TRAIL-mediated DR5-DISC formation by decoy receptors 1 and 2. Mol Cell Biol 26: 7046-7055, 2006.

22. Busch C, Jacob C, Anwar A, et al: Diallylpolysulfides induce growth arrest and apoptosis. Int J Oncol 36: 743-749, 2010.

23. Lee BC, Park BH, Kim SY and Lee YJ: Role of Bim in diallyl trisulfide-induced cytotoxicity in human cancer cells. J Cell Biochem 112: 118-127, 2011.

24. Balch CM, Buzzaid AC, Soong SJ, et al: Final version of the American Joint Committee on Cancer staging system for cutaneous melanoma. J Clin Oncol 19: 3635-3648, 2001.

25. Nakagawa $\mathrm{T}$, Zhu H, Morishima N, et al: Caspase-12 mediates endoplasmic-reticulum-specific apoptosis and cytotoxicity by amyloid-beta. Nature 403: 98-103, 2000.

26. Szegezdi E, Fitzgerald U and Samali A: Caspase-12 and ER-stress-mediated apoptosis: the story so far. Ann NY Acad Sci 1010: 186-194, 2003.

27. Rutkowski DT and Kaufman RJ: A trip to the ER: coping with stress. Trends Cell Biol 14: 20-28, 2004.

28. Hersey P and Zhang XD: Adaptation to ER stress as a driver of malignancy and resistance to therapy in human melanoma. Pigment Cell Melanoma Res 21: 358-367, 2008.

29. Liu H, Jiang CC, Lavis CJ, et al: 2-Deoxy-D-glucose enhances TRAIL-induced apoptosis in human melanoma cells through XBP-1-mediated up-regulation of TRAIL-R2. Mol Cancer 8: $122,2009$.

30. Ferri KF and Kroemer G: Organelle-specific initiation of cell death pathways. Nat Cell Biol 3: E255-E263, 2001. 
31. Rao RV, Castro-Obregon S, Frankowski H, et al: Coupling endoplasmic reticulum stress to the cell death program. An Apaf-1-independent intrinsic pathway. J Biol Chem 277: 21836-21842, 2002.

32. Morishima N, Nakanishi K, Takenouchi H, Shibata T and Yasuhiko Y: An endoplasmic reticulum stress-specific caspase cascade in apoptosis. Cytochrome c-independent activation of caspase- 9 by caspase-12. J Biol Chem 277: 34287-34294, 2002.

33. Xie Q, Khaoustov VI, Chung CC, et al: Effect of tauroursodeoxycholic acid on endoplasmic reticulum stress-induced caspase-12 activation. Hepatology 36: 592-601, 2002.
34. Mandic A, Hansson J, Linder S and Shoshan MC: Cisplatin induces endoplasmic reticulum stress and nucleus-independent apoptotic signaling. J Biol Chem 278: 9100-9106, 2003.

35. Shellman YG, Howe WR, Miller LA, et al: Hyperthermia induces endoplasmic reticulum-mediated apoptosis in melanoma and non-melanoma skin cancer cells. J Invest Dermatol 128: 949-956, 2008. 